\title{
Trends in glioblastoma treatment research: an analysis of clinical trials and literature
}

\author{
Aleksander E. Łaba ${ }^{1,2}$, Piotr Ziółkowski ${ }^{1}$ \\ ${ }^{1}$ Department of Pathology, Wroclaw Medical University, Wroclaw, Poland \\ ${ }^{2}$ Department of Neurology, Regional Specialist Hospital, Wroclaw, Poland
}

\begin{abstract}
Introduction. Glioblastoma is the most common, and the most lethal, primary malignant brain tumour in adults. The aim of the study was to present a comprehensive, data-based review of glioblastoma treatment research, considering all clinical trials and peer-reviewed journal publications.

Materials and methods. Data regarding all glioblastoma clinical trials that was available on 7 August 2019 on ClinicalTrials.gov was analysed. Information on interventions' mechanisms of action was obtained from AdisInsight. A PubMed search for'glioblastoma' was performed in September 2019. Citation counts were gathered from Scopus. Custom software for obtaining and analyzing data was developed by the authors.

Results. 1,388 clinical trials on glioblastoma with a start date between 1979 and 2020 were identified. The distribution of glioblastoma clinical trial phases differs significantly from that of other high-mortality cancers. 526 unique interventions of clinical trials and 206 molecular targets have been isolated. 32,410 publications on glioblastoma have been found, the number having increased especially since 2006. Publications on identified treatment options comprised $32.2 \%$. Publications on glioblastoma are cited on average 4.27 times per year. The average specificity of treatment options' publications for glioblastoma is $6.9 \%$.

Conclusions. Glioblastoma treatment options and their molecular targets can be quantitatively ranked according to their scientific research output. To the best of our knowledge, no such registries have been elaborated before.
\end{abstract}

Key words: glioblastoma, treatment, research, clinical trial, literature, review

(Neurol Neurochir Pol 2021; 55 (3): 269-280)

\section{Introduction}

Glioblastoma is the most common, and the most lethal, primary malignant brain tumour in adults. Each year, more than 3 per 100,000 people in the United States [1], Europe [2-4], Israel [5], Australia [6] and elsewhere are diagnosed with this WHO grade IV glioma, especially so in areas of higher socioeconomic status $[7,8]$. Although there are regions with a lower incidence, such as Japan, Korea $[9,10]$ and Jordan [11], the reason for this is not yet fully understood. The overall incidence is increasing $[4,9,12]$, yet this has not been convincingly attributed either to growing mobile phone use $[13,14]$, nor to improved diagnostic techniques [15], nor merely to the ageing of society.

The tumour manifests itself predominantly in patients aged 55-85 [1], with a median age at diagnosis of 63 [12]. Incidence rates increase with age up to $80-85$ years $[12,16]$. WHO-recognised [17] risk factors include: risk increase after exposure to ionising radiation to the head and neck, and risk decrease with history of allergies and atopic disease [14]. Additionally, the diagnosis is not always straightforward and swift, as symptoms are often vague, including headache (27\%), fatigue (20\%), confusion (27\%), and drowsiness (35\%), before progressing to more neurologically distinctive ones such as

Address for correspondence: Aleksander E. Łaba, Department of Pathology, Wroclaw Medical University, K. Marcinkowskiego 1 Str., 50-368 Wroclaw, Poland, e-mail: viamedica@drlaba.pl 
seizures (37\%) and motor deficits (21\%) [18]. Furthermore, the disease progresses quickly: Stensjøen et al. observed daily untreated tumour growth of $1.4 \%$ [19].

Consequently, the rapidly growing glioblastoma causes an enormous psycho-socioeconomic burden [20] to its often working-age and unsuspecting victims and their families.

The prognosis is highly unfavourable, with a five-year survival of no more than 5\% [21]. This makes glioblastoma one of the most deadly cancers $[22,23]$. Accordingly, survival is commonly given in months instead of years.

Palliative treatment (mostly with corticosteroids and anticonvulsants) offers survival ranging from a few weeks to a few months [21]. Decades of research on surgical resection and radiotherapy have prolonged this to about $6-10$ and 12 months respectively [21]. The 2005 introduction of concomitant and adjuvant temozolomide chemotherapy further increased survival by 2.5 months [24]. Refinement of these interventions has resulted in a current median survival of 16 months [25]. The remaining most frequently used treatment options include nitrosoureas (such as lomustine and carmustine), bevacizumab [26] and tumour treating fields [27]. The outcomes are especially promising for the latter, with reports of expanding median overall survival to almost 21 months [28].

Despite these encouraging new treatment modalities emerging just since 2005, the results are far from satisfactory, given that there is an average of more than 20 years of life lost due to premature mortality caused by glioblastoma [29]. Further research is highly desirable.

Over time, scientific research has provided us with significant advances in our understanding of the histopathological characteristics of glioblastoma. In 1926, 62 years after Rudolf Virchow coined the term 'glioma', Percival Bailey and Harvey Cushing were able to distinguish 'glioblastoma multiforme' (GBM) as a separate entity, although the name acknowledged the variable gross appearance of the tumour [30]. Nowadays, glioblastoma is still classified as a high-grade glioma with predominant diffuse astrocytic differentiation exhibiting hypercellularity, nuclear atypia, mitotic activity with microvascular proliferation and/or tumour necrosis [31]. Although the histopathology of the tumour remains extremely variable, with abundant cellular and nuclear polymorphisms alongside significant regional heterogeneity, the recent discoveries of molecular mechanisms and genetic alterations established the basis for the 2016 fourth edition of the WHO Classification of Tumours of the Central Nervous System [17] (Fig. S1, see supplementary materials). This changed the way we perceive glioblastoma. The discoveries are so profound that after 90 years we now see a new shift in nomenclature. The popularity of the broad term 'glioblastoma multiforme' has waned, giving way to more specific entities such as IDH-wildtype glioblastoma, giant cell glioblastoma, gliosarcoma, epithelioid glioblastoma, IDH-mutant glioblastoma, etc. [32] (Fig. S2, see supplementary materials). Despite the limited therapeutic success, it would be unfair to say that glioblastoma treatment research is scarce.
On the contrary: plentiful clinical trials are conducted [33-36] and articles published [37].

In fact, the amount of research is so abundant that it is challenging for any non-expert to grasp the glioblastoma therapeutic interventions outlook without becoming confused [38]. The scientific community has recently recognised this problem, and summaries of past studies are beginning to emerge. In 2012, Nieder et al. [37] analysed patterns of citations and reviewed articles published on glioblastoma between 2006 and 2010. They showed glioblastoma research activity increasing over time. The Journal of Clinical Oncology and the Journal of Neuro-Oncology were the major scientific journals in the field. Among the top 10 cited articles, seven reported on genomic analyses, molecular subclasses or stem cells, with only two articles on phase II or III clinical trials. In 2017, Cihoric et al. [34] analysed 2005-2015 phase II and III clinical trials, providing insight into, inter alia: experimental interventions, clinical trials' funding, enrollment, and phase distribution. They found a high initiation of glioblastoma clinical trials, suggesting that this was caused by the failure of previous early investigative treatments to show satisfactory efficacy. The authors found that $51.9 \%$ of trials were funded primarily by the industry, consistent with other oncology clinical trials. They demonstrated, however, insufficient representation of surgery, radiotherapy, and imaging focused trials, with these funded solely by academic institutions. At the time, the most researched topics were treatment options targeting EGFR or VEGF receptors and their pathways, as well as multi-TKIs. In 2018, Vanderbeek et al. [35] reviewed interventional glioblastoma clinical trials initiated between 2005 and 2016, analysing, inter alia: clinical trials' duration, enrollment, phase distribution, selected endpoints, and connected publications as linked by ClinicalTrials.gov. The authors found that a significant minority of glioblastoma patients were enrolled in clinical trials, although this observation did not differ much from general oncology trials. They showed long development times to be characteristic of glioblastoma research, and suggested suboptimal decision making that led to too many patients with ineffective therapies engaging significant financial resources. Also in 2018, Paolillo et al. [33] reviewed 2015-2017 clinical trials and selected therapeutic strategies. They reported multiplying strategies against glioblastoma and a growing knowledge of genetic profiling and mutations. The authors considered new immunotherapy strategies to show the most promise. They stressed, however, that traditional therapies such as surgery, chemotherapy, and radiation remain the first line approaches to glioblastoma. In 2019, Zanders et al. [36] reviewed the 2016-2018 glioblastoma clinical trials. They concluded that small-molecule interventions have not significantly improved the standard of care, pointing out, nonetheless, that the vast majority of new clinical trials continue to focus on small-molecule therapy. They suggested however that together with smarter combination therapy selection and adaptive clinical trial design, 
small-molecule interventions could still show success. These authors felt the best hope lay in novel immunotherapies and developments in the modulation of $\mathrm{T}$ cells.

The aim of the current study is to present a comprehensive, data-based review of glioblastoma treatment research, considering all past clinical trials and scientific journal publications. This article is intended to serve as a roadmap for anyone interested in the subject, and as a guide for further research in the pursuit of effective glioblastoma treatment.

\section{Methods}

\section{Clinical trials}

\section{Glioblastoma}

Complete XML data for all clinical trials found by searching for the condition of 'glioblastoma' was downloaded from ClinicalTrials.gov on 7 August 2019. Sought after information was extracted and saved: this included data on clinical trial status, phase, enrollment, intervention(s), study type, start, and completion dates. Calculations in the current study involved only clinical trials started after 1993, due to incomplete data for older entries.

\section{Other cancers}

Analogous information about clinical trials was gathered for cancers that had data on incidence, mortality, and five-year survival rate available on the National Cancer Institute's Surveillance, Epidemiology, and End Results (SEER) Programme website [23]. The cancers selected are listed in Table S1 (see supplementary materials). This data was used to contrast and compare glioblastoma to other cancers.

\section{Treatment options}

\section{Inclusion and exclusion criteria for interventions of glioblastoma clinical trials}

Diagnostic interventions were intentionally excluded as they were not the subject of this study. Due to the automatic processing of large amounts of data, we included interventions with consistently identifiable nomenclature. Interventions with names that were not specific enough (e.g. 'surgery', 'radiation therapy') were excluded. Interventions with synonymous names listed as separate entities on ClinicalTrials.gov were merged. Combination interventions were divided into single entities. Interventions that were included in this study are summarised in Table S2 (see Supplementary materials).

\section{Treatment options in scientific literature}

We searched PubMed [39] for articles on the treatment of glioblastoma published from 2010 onwards. The search was conducted on 16 February 2019 with a query listed in Appendix 1 . Search results were imported to Mendeley Reference
Management Software. Glioblastoma treatment options were extracted by reading through the titles and abstracts of the publications found.

\section{Alternative names and categorisation}

The National Cancer Institute Thesaurus [40] and Springer AdisInsight [41] were searched for synonyms of treatment options. A comprehensive list of alternative names (i.e. synonyms) warranted the reliability of further automated literature analysis, and was therefore paid much attention.

AdisInsight [41] was also used for categorisation of treatment options according to their 'mechanism of action' and (drug) 'class'. We then combined the mechanisms of action into broader categories based on molecular targets of treatment options. The aim of this approach was to more clearly present numerous results.

\section{Literature}

PubMed [39] was selected as a source for data on glioblastoma research [42]. It was searched for each of the identified glioblastoma treatment options in September 2019. Search queries included previously gathered synonymous names. They were targeted to glioblastoma. The results were downloaded in XML format and parsed. Extracted information included publication identifiers (DOI, PMID), publication dates, journal, and publication type.

Accordingly, on 22 September 2019, the authors once again searched PubMed with queries for each of the identified treatment options. These queries were however targeted to neoplasms in general. They, too, included treatment options' alternative names. Total number of publications found and their distribution by year were saved for each treatment option.

Finally, a large-scale PubMed search was performed for the entire field of glioblastoma research on 29 September 2019 [39] to serve as a baseline for further analysis. Publication identifiers, dates, journals, affiliations, and types were saved.

All search queries used are listed in Appendix 1.

\section{Glioblastoma specificity}

Research specificity to glioblastoma was calculated for each treatment option as a percentage of publications on glioblastoma to all publications on neoplasms in general (as explained in the previous section).

\section{Distribution of studies by country}

Country information was extracted from the affiliations of glioblastoma publications gathered from PubMed.

\section{Citation analysis}

Citation counts for all publications on glioblastoma (including publications on identified glioblastoma treatment options) were gathered. Scopus [43] was selected as the most suitable source for citation data. This was primarily due to its advantageous coverage of newer (i.e. after 1995) publications 
[42] that were deemed more valuable for the current study than older ones. In addition, the authors recognised Scopus's advantage over Google Scholar and Web of Science in terms of website architecture and suitability for automated data collection. Given the large amount of data to gather (citation counts for over 30,000 publications), the process took six days.

\section{Other cancers}

Data on the number of publications on cancers selected from the SEER Programme website [23] (Tab. S1, see supplementary materials), and their distribution by year, was downloaded from PubMed.

\section{Glioblastoma treatment research rating}

The authors created an original algorithm for rating glioblastoma treatment options based on their research data. Treatment options were ordered by quantitative criteria and then ranked according to the sum of their relative positions in each criterion. These criteria consisted of: 1 ) the number of clinical trials conducted, 2) total enrollment, 3) average number of publications per year, 4) average citation count per publication per year, 5) specificity of publications to glioblastoma, and 6) year of introduction in a clinical trial, favouring newer treatments because past treatments have shown limited efficacy despite more time for fine-tuning the regimes. The items were ordered in descending order for all the criteria.

Mechanisms of action and their common molecular targets were selected in the same fashion, with an additional criterion of: 7) number of treatment options in each category, likewise in descending order.

\section{Data gathering}

All steps of data gathering were accompanied with C\#.NET applications that saved the data in appropriate SQLite databases. This tailor-made software was developed by the authors.

\section{Statistical analysis}

Basic descriptive analysis was carried out programming directly in SQL. The remaining descriptive, and all regression, analysis was performed in $\mathrm{R}$ programming language. The 'segmented' $R$ package [44] was used for change-point regression (Fig. S2, S5, S7, see supplementary materials). Obtaining p-values from confidence intervals was accomplished using the method proposed by Altman and Bland [45].

\section{Results}

\section{Clinical trials}

1,388 clinical trials on glioblastoma were identified, with start years ranging between 1979 and 2020. There has been a constant increase of almost four additional new trials started each year $\left(R^{2}=0.94, p<0.001\right)$ since 1990 (Fig. S3, see supplementary materials). This number now exceeds 90 yearly trials. The vast majority (91.6\%) of clinical trials are of interventional study type, although there has been a slight decrease over time in favour of observational clinical trials $(\mathrm{p}<0.01)$.

There are currently 379 (27.3\%) active (recruiting, not recruiting or enrolling by invitation) clinical trials with completion dates after August 2019. Their enrollment totals 45,225. Early phase I (phase 0 ) trials comprise $16(4.2 \%)$ trials, phase I - 168 (44.3\%) trials, phase II - 164 (43.3\%) trials, phase III $-23(6.1 \%)$ trials, and phase IV - one $(0.3 \%)$ trial. The distribution of phases in currently active clinical trials does not differ statistically significantly $(\mathrm{p}=0.17)$ from the distribution of all-time interventional glioblastoma clinical trials (Fig. S4 B, see Supplementary materials). There is also no statistically significant annual change in the relative distribution of glioblastoma clinical trial phases. Nevertheless, each year, more clinical trial interventions are tested in phase I (increasing by 2.8 per year, $\mathrm{p}<0.001, \mathrm{R}^{2}=0.83$ ) and phase II (increasing by 3.0 per year, $\left.p<0.001, R^{2}=0.78\right)$ clinical trials. There seems to be also a moderate increase (by 0.3 per year, $\mathrm{p}<0.05$, $\mathrm{R}^{2}=0.39$ ) in interventions tested in phase IV trials (Fig. S4 C, see supplementary materials). No statistically significant increase was found for phase III $\left(\mathrm{p}=0.07, \mathrm{R}^{2}=0.18\right)$.

The distribution of glioblastoma clinical trial phases differs significantly from that of clinical trials for other examined high-mortality cancers $(\mathrm{p}<0.001)$ (Fig. S4 A, see Supplementary materials). However, no statistically significant correlation between phase distribution and five-year survival or mortality rates was observed for the analysed 30 neoplasms.

\section{Treatment options}

1,583 unique entries for interventions of clinical trials have been identified. ClinicalTrials.gov categorises interventions into 11 categories (Tab. S2, see supplementary materials). 'Other' interventions were largely composed of neuroradiological (e.g. 'MRI'), diagnostic (e.g. 'blood draw'), and research (e.g. 'questionnaire'). 'Procedure' category contained mostly surgical and diagnostic interventions. 'Device' interventions included many neurodiagnostic devices. 'Genetic' interventions were almost exclusively laboratory techniques (e.g. 'DNA analysis', 'polymorphism analysis'). 'Behavioural' category predominantly comprised activities such as 'psychoeducation' and 'exercise'.

526 unique interventions of clinical trials have been isolated after data clean-up according to the procedure described in the Methods section. These interventions were undertaken 2,072 times in clinical trials. Each intervention was tested, on average, in 3.94 clinical trials $(S D=16.77)$. However, both the median and the mode are equal to 1 , as more than half of the interventions were tested in single trials $(278 / 526=52.8 \%)$.

\section{Treatment options in scientific literature}

1,487 publications were retrieved from the search on glioblastoma treatment. Investigation of the publications allowed for the identification of 181 treatment options. 172 of them 
were listed as interventions in clinical trials. Not tested in clinical trials were nine treatment options (two nutritional and seven drugs). A total of $535(526+9)$ glioblastoma treatment options further analysed in this study are summarised in Table S2 (see Supplementary materials).

\section{Alternative names and categorisation}

4,452 alternative names were identified for 515 (96.2\%) treatment options, with an average of 8.64 names per treatment option ( $\mathrm{SD}=7.65$ ). No synonyms were found for the remaining 20 treatment options, mostly immunological (10/20, e.g. Ad-hCMV-TK) and radiotherapeutic (4/20).

427 mechanisms of action were found for 503 (94.0\%) treatment options. There was an average of 2.34 mechanisms of action per treatment option ( $S D=1.95)$, and 2.75 treatment options per mechanism of action $(S D=5.04$, median $=1$ ). Molecular target-based categorisation allowed for a reduction of category number to 206. 407 (drug) classes were identified for 486 treatment options, with an average of 4.13 classes per treatment option ( $\mathrm{SD}=2.35$ ), and $4.93(\mathrm{SD}=21.8$, median $=2)$ treatment options per class. Mechanisms of action and classes were unavailable in AdisInsight for radiotherapeutic and surgical treatment modalities as well as for therapeutic devices (e.g. Optune) and dietary supplements.

\section{Novel treatment options}

There have been, on average, 18 novel interventions introduced annually in clinical trials $(\mathrm{SD}=11$, median $=18)$. This number increases each year by an average of 1.2 more novel treatment options, and currently exceeds 30 (Fig. 1). Every year, there are new mechanisms of action introduced as well as new common molecular targets. The latter, however, show a somewhat steadier annual rate (Fig. 1).

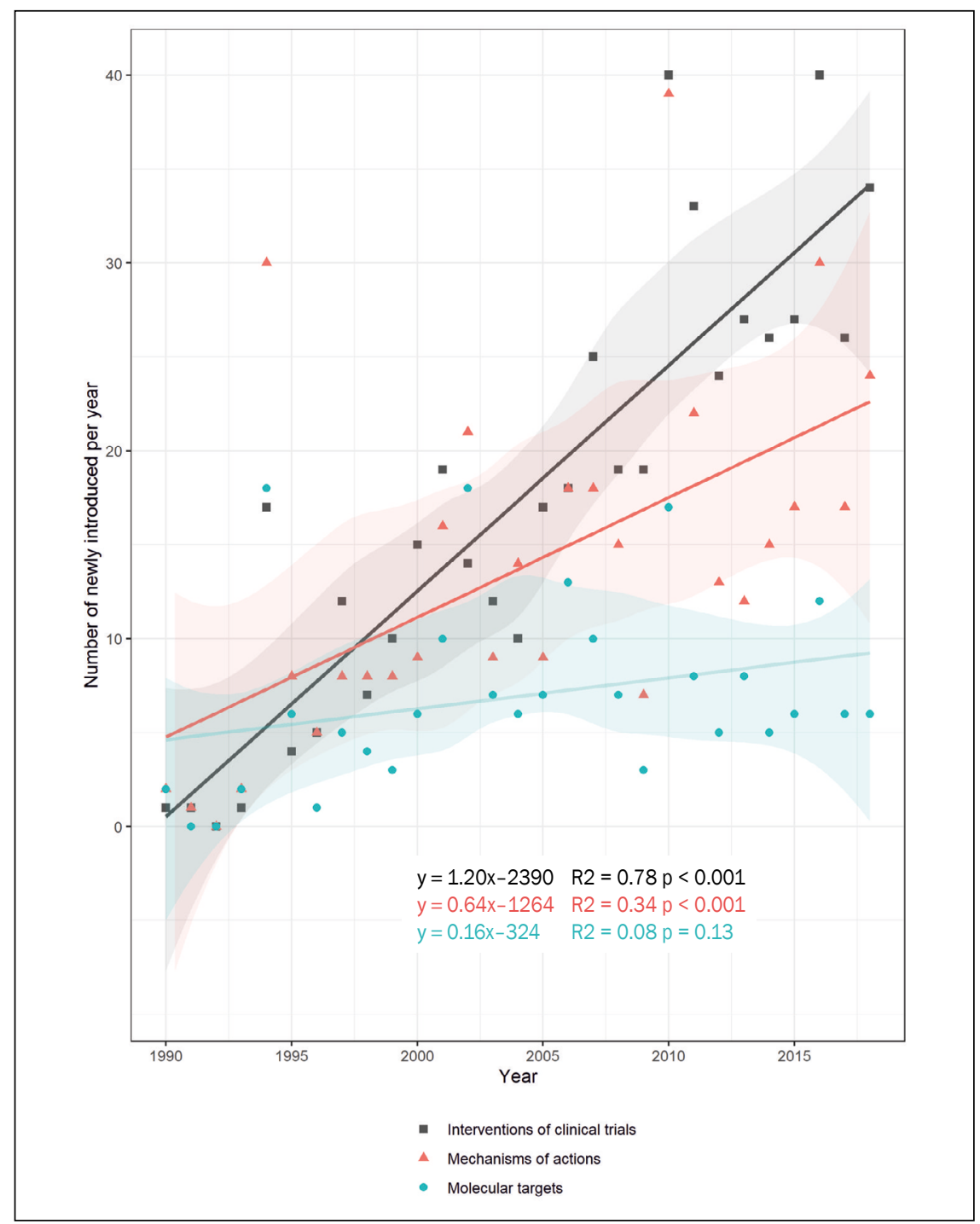

Figure 1. New treatment options, mechanisms of action, and molecular targets by year of first introduction in clinical trials 


\section{Literature}

32,410 publications on glioblastoma have been identified. The number of publications is increasing each year and has especially accelerated since 2006 (Fig. S5 A, see supplementary materials). A summary of the most popular publication types is presented in Table S3 (see supplementary materials). Most papers on glioblastoma have been published in the journals listed in Table S4 (see supplementary materials). Similarly to that shown by Nieder et. al in 2012 [37], both the Journal of Clinical Oncology and the Journal of Neuro-Oncology continue to contain the highest numbers of glioblastoma articles. The latter, however, is approached by PloS one, Oncotarget and Cancer research (see Table S4 in supplementary materials).

10,435 publications on treatment options were found. This number increases each year and has especially accelerated since 2004. Publications on identified treatment options comprise $32.2 \%$ of all glioblastoma research. This percentage has increased statistically significantly $(p<0.001)$ since 1961 at an average yearly rate of $0.69 \%$ (Fig. S6, see supplementary materials). No publications were found for 129 treatment options. Each of the remaining 406 treatment options was presented in an average of 39 publications, although the median was equal to 7 , mode to 1 , and standard deviation was 196 publications. Treatment options are mentioned in publications targeted to glioblastoma, on average, 14.2 years $(\mathrm{SD}=14.6$ years, median $=8$ years) after they were first mentioned in publications targeted to neoplasms in general. However, there seems to be a tendency towards shortening this timespan over time, especially after 1980, at an average rate of 0.29 years difference per year ( $<0.001)$ (Fig. S7, see supplementary materials).

Publication preceded clinical trial in the case of 200 treatment options (on average by 10.9 years, $\mathrm{SD}=10.8$, median $=7$ years). In 161 cases, publications appeared after the first clinical trial started (on average after 3.7 years, $\mathrm{SD}=3.2$, median $=3$ years). In 32 cases, the first clinical trial started in the same year as the first publication (Fig. S8, see supplementary materials). There is no statistically significant change over time of the overall average timespan between the first clinical trial and the first publication $(\mathrm{p}=0.61)$. There seems to be, however, a tendency to shorten this timespan in the clinical-trial-first group by an average of 0.2 years per year $\left(\mathrm{R}^{2}=0.14, \mathrm{p}<0.001\right)$.

\section{Glioblastoma specificity}

Average specificity of publications on treatment options for glioblastoma is $6.9 \%(\mathrm{SD}=16.0 \%$, median $=1.5 \%)$. There is no statistically significant trend for change over time when treatment options are analysed according to their clinical trial introduction year $\left(\mathrm{p}=0.88, \mathrm{R}^{2}<0.01\right)$.

\section{Distribution of studies by country}

A world map with the numbers of cumulative glioblastoma articles (co)authored in given countries is presented in Fig. S9 (see supplementary materials). Over $81 \%$ of all glioblastoma articles have been published in just $11 \%$ of countries $(n=16)$.
Moreover, articles from just seven countries (USA, China, Germany, Japan, Italy, France, and Canada) represent over $53 \%$ of all the glioblastoma articles available on PubMed. The percentage shares of articles on the analysed treatment options relative to all glioblastoma articles remain fairly constant across countries: they are approximately normally distributed with a mean of $34 \%(S D=5 \%)$.

An up-to-date world map showing the numbers of glioblastoma clinical trials is available online at https://clinicaltrials.gov/ct2/results $/$ map?cond=Glioblastoma

\section{Citation analysis}

Citation data was available in Scopus for 30,267 publications on glioblastoma (94\%). There is an average of 4.27 citations per publication per year $(\mathrm{SD}=11.85$, median $=2.08$ ).

9,855 publications on treatment options for glioblastoma $(94,4 \%)$ had citation data available in the Scopus database. There are statistically significantly $(\mathrm{p}<0.05)$ more citations per publication per year in the publications on the treatment options group than in all publications on the glioblastoma group, an average for the former being $4.73(\mathrm{SD}=12.04)$ with a median of 2.50 citations.

Changes over time of citations per publication per year of publications on glioblastoma and on treatment options in glioblastoma are presented in Figure 2.

It is possible that publications on glioblastoma generally favour the growth of journals' impact factor, as the overall yearly citation effect is approaching its impact factor multiplied by 1.5 [46] (Tab. S4). However, a separate study would be needed for conclusive results. Factors to be taken into account should include, at least, publication type (article/ /review), yearly citations separated since publication, and yearly journal impact factor.

Of the top 10 articles with the most citations per year (Tab. S5, see supplementary materials), the majority report on glioblastoma characteristics rather than treatment, similarly to how it was described by Nieder et al. in their 2012 review [37].

\section{Glioblastoma treatment research rating}

The top 30 treatment options selected according to the proposed algorithm are presented in Table 1. Molecular targets of the most researched treatment options are summarised in Table 2.

\section{Limitations}

Despite great attention to detail, planning and organisation, the authors were unable to eliminate all limitations.

\section{Clinical trials}

The authors' aspiration not to omit treatment options by limiting the study to subjective selection has, at times, led to overinclusion and posed a threat of overestimation. For example, the clinical trial NCT04028479 investigating chimeric antigen receptor T-cell therapy in 76 cancers (including glioblastoma) must have been manually excluded from the 


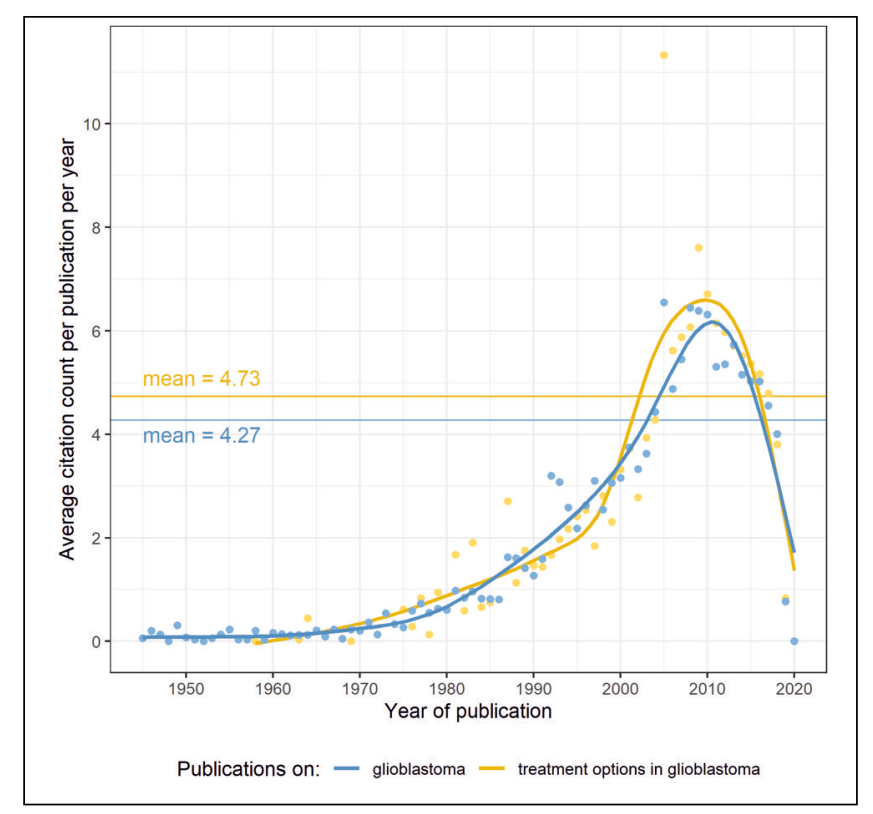

Figure 2. Average citations of publications on glioblastoma and on glioblastoma treatment options

calculations of total enrollment due to the outlined value (enrollment of 100,000 when there is a total enrollment for all the remaining glioblastoma trials of 104,752). But not including this trial altogether because it is not glioblastoma-specific (as practiced by some authors) would however in itself induce bias by underestimating CAR-T research in glioblastoma. Although great attention was given to identifying and correcting any instances of such bias, some may have escaped our scrutiny.

\section{Alternative names and categorisation of interventions}

There is no one ideal way to categorise treatment options according to their mechanisms of action. The approach of presenting them in narrow categories, such as listed in the AdisInsight database, risks underestimation. For example, drugs targeting topoisomerase enzymes are listed under separate categories of type I and type II inhibitors. When the number of drugs is calculated independently for each category, topoisomerase is the $27^{\text {th }}$ most numerous treatment target. When, however, both mechanisms of action are regarded as one category of topoisomerase-targeting drugs, topoisomerase becomes the $16^{\text {th }}$ most numerous target. The authors believe the latter approach more faithfully represents the whole picture of glioblastoma treatment research, but we nonetheless must warn the reader of the risk of overestimation.

\section{Epidemiological statistics}

Readers should be warned that Table S2 (see supplementary materials) may be biased since, due to the limited epidemiological data availability, a rather specific entity of glioblastoma is compared to broader disease definitions like breast or lung cancer (instead of e.g. small-cell lung cancer). However, the observation that there are more clinical trials on brain cancers than would be expected from the disease incidence is consistent with the results of Hirsch et al. [47]. Our intention was to illustrate the noninferiority of glioblastoma research compared to other neoplasms, and also the ones of higher incidence.

\section{Distribution of studies by country}

Data for the analysis was gathered by means of a PubMed search, as explained in the Methods section. This may understate the number of articles from countries other than the USA and/or not written in English, as there is research published in local scientific journals not (fully) indexed on PubMed [48].

\section{Conclusions}

Glioblastoma treatment options can be quantitatively ranked according to their scientific research output, as presented in Table 1. Similarly, molecular targets can be ordered (Tab. 2). To the best of our knowledge, no such registries have been elaborated before.

Additionally, to the best of our knowledge, this study is the first to have conducted an essential bibliometric analysis on all PubMed-found glioblastoma-related publications. Publications on glioblastoma are expected to be cited on average 4.27 times per year. Publications regarding treatment options are expected to be cited slightly more often (on average 4.73 times per year). Although further research would be desirable, these results suggest that journals may welcome publications on glioblastoma not only for their readability, but also for their citation advantage. 


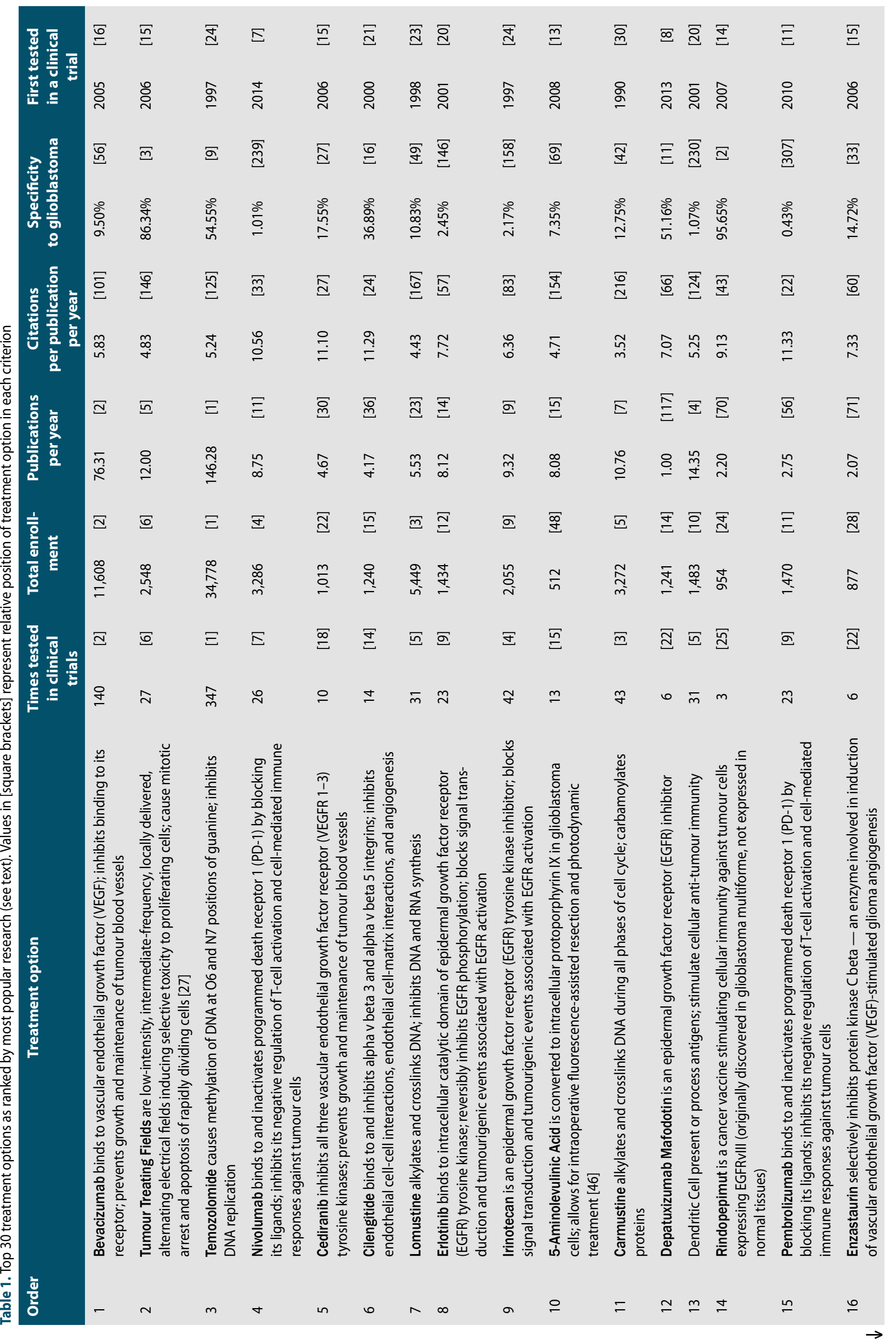




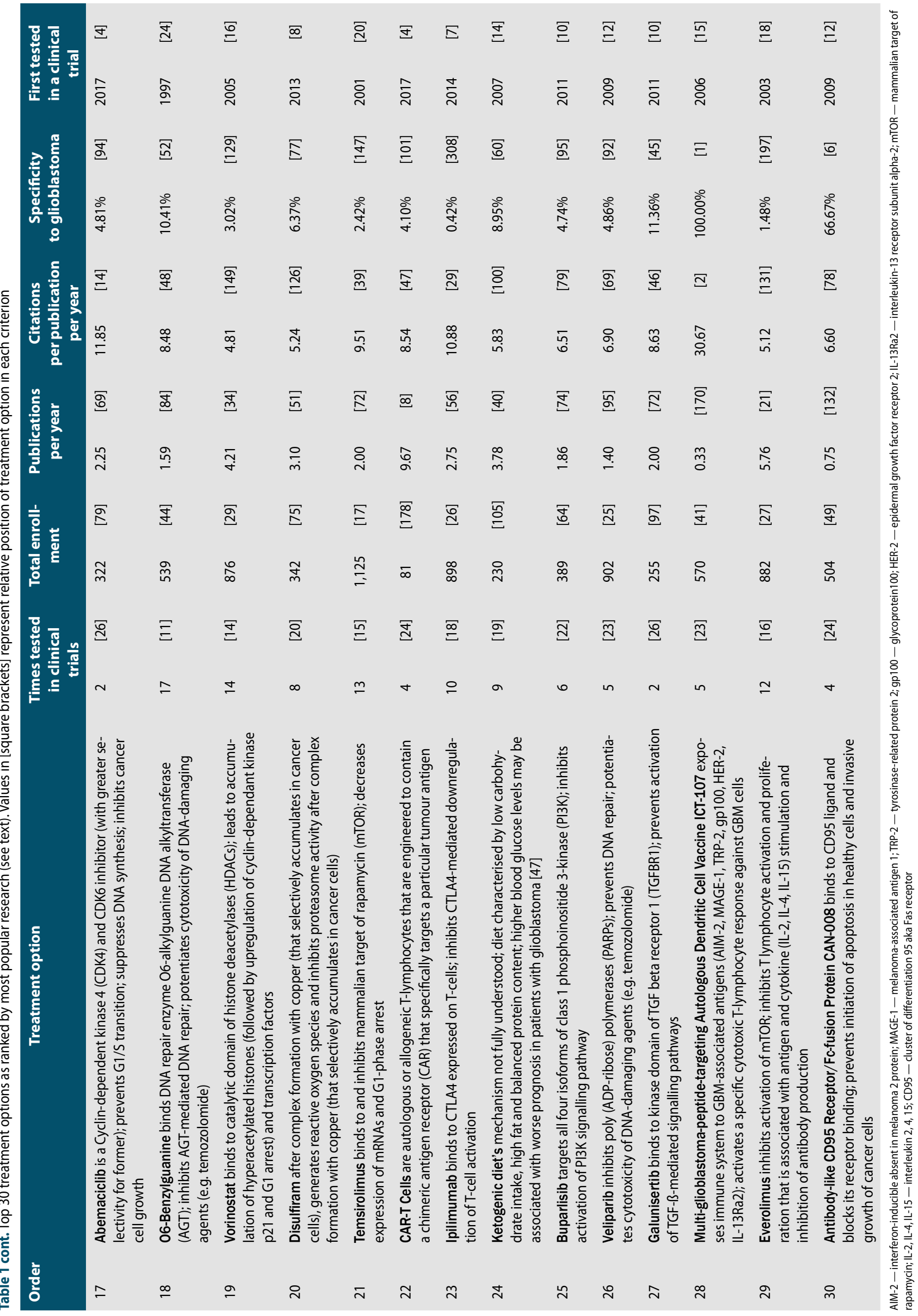




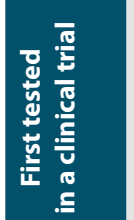

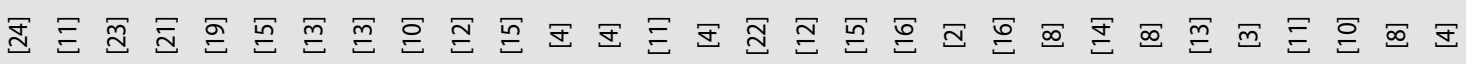

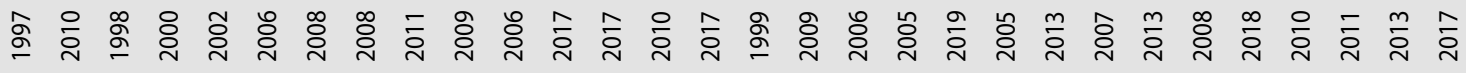

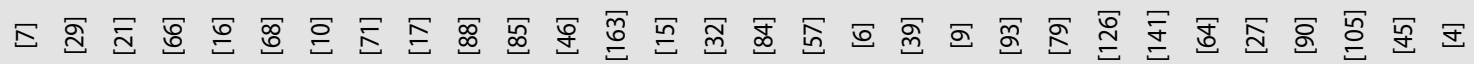

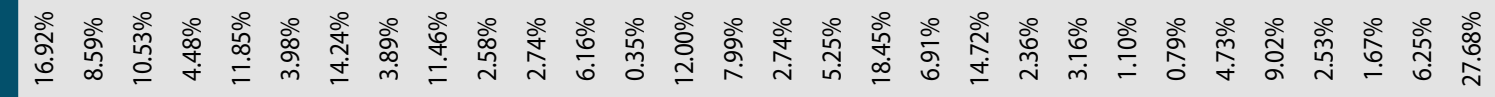

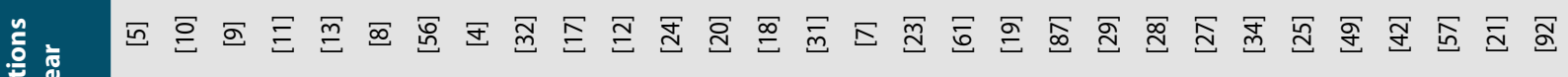

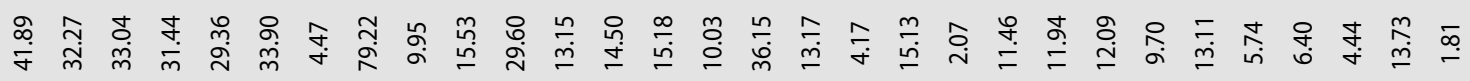
๘

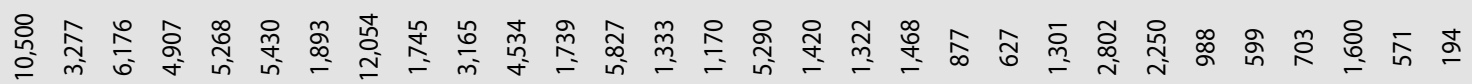

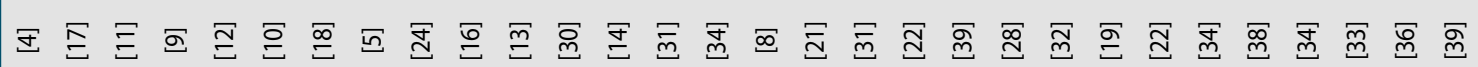

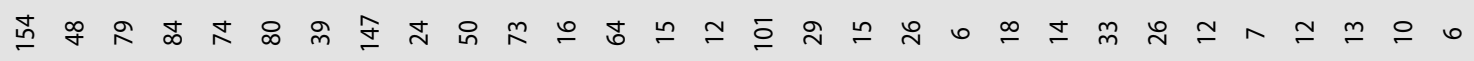

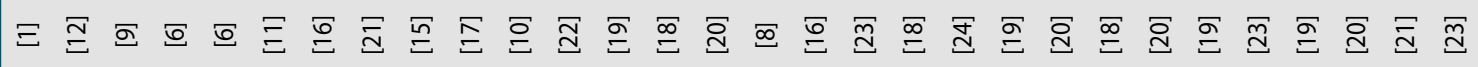
蒙

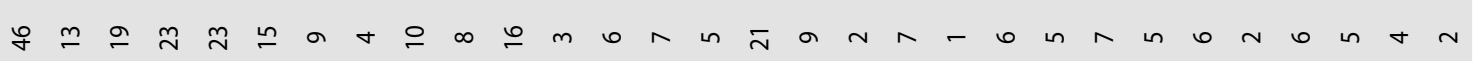

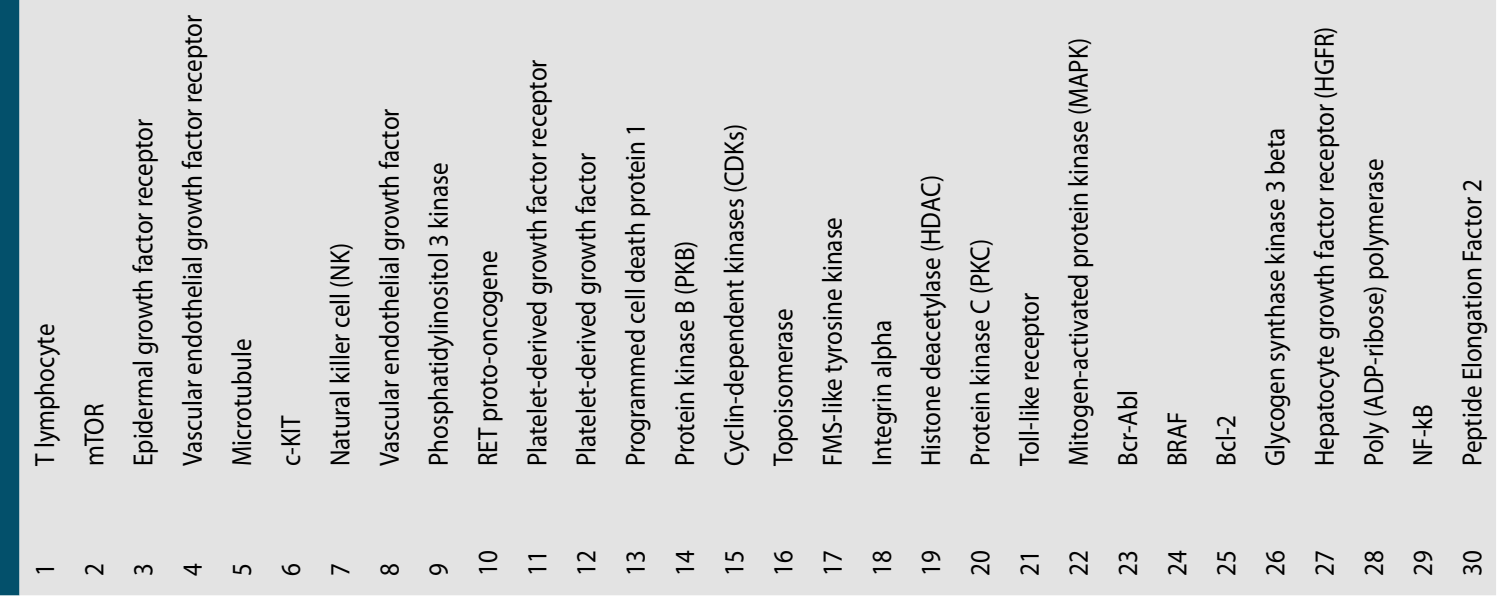


Scientists' interest in publishing on glioblastoma has been increasing significantly since 2006, possibly due to the encouraging 2005 introduction of the Stupp protocol of temozolomide treatment [24] (Fig. S5 A, see supplementary materials). It is interesting to note, however, that clinical trials have not followed that trend, with a rather steady annual increase rate (Fig. S3, see supplementary materials).

Nonetheless, the number of clinical trials on glioblastoma is constantly growing at a relative increase rate not inferior to that of other cancers. The best predictor for clinical trial number seems to be the cancer incidence rate. Five-year survival rates help to better explain clinical trial count, with more trials for cancers with shorter survival. Cancer prevalence in the USA is only a weak predictor of research output. Cancer publication count is strongly positively correlated with clinical trial count. It, too, depends on cancer incidence and fiveyear survival rate. Marginally more than half of glioblastoma treatment options have had glioblastoma-targeted scientific publications before having a clinical trial conducted. This observation remains constant over time.

The number of new glioblastoma treatment options as well as new mechanisms of action is rapidly growing each year. However, the specificity of their publications for glioblastoma is low, with no clear trend of increase over time. The results reveal also that interventions are tested in glioblastoma, on average, 10 years after they were first published on other neoplasms. More than half of clinical trial interventions have only been tested in single trials. The vast majority was tested in earlier phase clinical trials, which is consistent with what Cihoric et al. observed in their 2017 review [34]. However, there seems to be a trend for a moderate increase in the number of glioblastoma treatment options studied in phase IV clinical trials, and no clear negative trend for phase III was demonstrated.

Searching ClinicalTrials.gov website is a faster, more convenient, and reliable way of gathering a comprehensive list of glioblastoma treatment options than analysing publications on PubMed. Given the strong correlation between clinical trials and publications for glioblastoma and other cancers, the same premise might hold true for the latter. However, data on glioblastoma clinical trials' interventions could be better organised on ClinicalTrials.gov. Categorisation into intervention types ('drug', 'device', etc.) in its current form cannot be used to reliably filter interventions. Separating treatment from dosage and maintaining a list of unique treatments would prevent categorising the same treatment as different entities. There have been successful attempts to overcome this issue by privately-run databases such as Springer's AdisInsight [41].

However, they usually demand a fee and, most importantly, still depend on data from ClinicalTrials.gov.

\section{References}

1. Ostrom QT, Gittleman H, Farah P, et al. CBTRUS statistical report: Primary brain and central nervous system tumors diagnosed in the
United States in 2006-2010. Neuro Oncol. 2013; 15 Suppl 2: ii1-i56, doi: 10.1093/neuonc/not151, indexed in Pubmed: 24137015.

2. Brodbelt A, Greenberg D, Winters T, et al. (UK) National Cancer Information Network Brain Tumour Group. Glioblastoma in England: 2007-2011. Eur J Cancer. 2015; 51(4): 533-542, doi: 10.1016/j. ejca.2014.12.014, indexed in Pubmed: 25661102.

3. Hansen S, Rasmussen BK, Laursen RJ, et al. Treatment and survival of glioblastoma patients in Denmark: The Danish Neuro-Oncology Registry 2009-2014. J Neurooncol. 2018; 139(2): 479-489, doi: 10.1007/s11060-018-2892-7, indexed in Pubmed: 29754199.

4. Natukka T, Raitanen J, Haapasalo $\mathrm{H}$, et al. Incidence trends of adult malignant brain tumors in Finland, 1990-2016. Acta Oncol. 2019; 58(7): 990-996, doi: 10.1080/0284186X.2019.1603396, indexed in Pubmed: 30985227.

5. Sadetzki S, Zach L, Chetrit A, et al. Epidemiology of gliomas in Israel: a nationwide study. Neuroepidemiology. 2008; 31(4): 264-269, doi: 10.1159/000165366, indexed in Pubmed: 18931523.

6. Dobes M, Khurana VG, Shadbolt B, et al. Increasing incidence of glioblastoma multiforme and meningioma, and decreasing incidence of Schwannoma (2000-2008): Findings of a multicenter Australian study. Surg Neurol Int. 2011; 2: 176, doi: 10.4103/2152-7806.90696, indexed in Pubmed: 22276231.

7. Cote DJ, Ostrom QT, Gittleman H, et al. Glioma incidence and survival variations by county-level socioeconomic measures. Cancer. 2019; 125(19): 3390-3400, doi: 10.1002/cncr.32328, indexed in Pubmed: 31206646.

8. Muquit S, Parks R, Basu S. Socio-economic characteristics of patients with glioblastoma multiforme. J Neurooncol. 2015; 125(2): 325-329, doi: 10.1007/s11060-015-1913-z, indexed in Pubmed: 26334316.

9. Miranda-Filho A, Piñeros M, Soerjomataram I, et al. Cancers of the brain and CNS: global patterns and trends in incidence. Neuro Oncol. 2017; 19(2): 270-280, indexed in Pubmed: 27571887.

10. Lee $\mathrm{CH}$, Jung $\mathrm{KW}$, Yoo $\mathrm{H}$, et al. Epidemiology of primary brain and central nervous system tumors in Korea. J Korean Neurosurg Soc. 2010; 48(2): 145-152, doi: 10.3340/jkns.2010.48.2.145, indexed in Pubmed: 20856664.

11. Tamimi AF, Tamimi I, Abdelaziz M, et al. Epidemiology of Malignant and Non-Malignant Primary Brain Tumors in Jordan. Neuroepidemiology. 2015; 45(2): 100-108, doi: 10.1159/000438926, indexed in Pubmed: 26314301.

12. Korja M, Raj R, Seppä K, et al. Glioblastoma survival is improving despite increasing incidence rates: a nationwide study between 2000 and 2013 in Finland. Neuro Oncol. 2019; 21(3): 370-379, doi: 10.1093/neuonc/noy164, indexed in Pubmed: 30312433.

13. Chapman S, Azizi L, Luo Q, et al. Has the incidence of brain cancer risen in Australia since the introduction of mobile phones 29 years ago? Cancer Epidemiol . 2016; 42: 199-205.

14. Ostrom QT, Bauchet L, Davis FG, et al. The epidemiology of glioma in adults: a "state of the science" review. Neuro Oncol. 2014; 16(7): 896-913, doi: 10.1093/neuonc/nou087, indexed in Pubmed: 24842956.

15. Philips A, Henshaw DL, Lamburn G, et al. Brain Tumours: Rise in Glioblastoma Multiforme Incidence in England 1995-2015 Suggests an Adverse Environmental or Lifestyle Factor. J Environ Public Health. 2018; 2018: 7910754, doi: 10.1155/2018/7910754, indexed in Pubmed: 30034480.

16. Young JS, Chmura SJ, Wainwright DA, et al. Management of glioblastoma in elderly patients. J Neurol Sci. 2017; 380: 250-255, doi: 10.1016/j.jns.2017.07.048, indexed in Pubmed: 28870580. 
17. Louis D, Ongaki H, Wiestler O, et al. The 2007 WHO Classification of Tumours of the Central Nervous System. Acta Neuropathologica. 2007; 114(2): 97-109, doi: 10.1007/s00401-007-0243-4.

18. IJzerman-Korevaar M, Snijders TJ, de Graeff A, et al. Prevalence of symptoms in glioma patients throughout the disease trajectory: a systematic review. J Neurooncol. 2018; 140(3): 485-496, doi: 10.1007/s11060-018-03015-9, indexed in Pubmed: 30377935.

19. Stensjøen AL, Solheim 0 , Kvistad KA, et al. Growth dynamics of untreated glioblastomas in vivo. Neuro Oncol. 2015; 17(10): 1402-1411, doi: 10.1093/neuonc/nov029, indexed in Pubmed: 25758748.

20. Starnoni D, Berthiller J, Idriceanu TM, et al. Returning to work after multimodal treatment in glioblastoma patients. Neurosurg Focus. 2018; 44(6): E17, doi: 10.3171/2018.3.FOCUS1819, indexed in Pubmed: 29852767.

21. Delgado-López PD, Corrales-García EM. Survival in glioblastoma: a review on the impact of treatment modalities. Clin Transl Oncol. 2016; 18(11): 1062-1071, doi: 10.1007/s12094-016-1497-x, indexed in Pubmed: 26960561.

22. Siegel R, Miller K, Jemal A. Cancer statistics, 2019. CA: A Cancer Journal for Clinicians. 2019; 69(1): 7-34, doi: 10.3322/caac.21551.

23. National Cancer Institute (NIH). SEER Cancer Statistics. https://seer. cancer.gov/statfacts/ (2019 Sep 8).

24. Stupp R, Mason WP, van den Bent MJ, et al. European Organisation for Research and Treatment of Cancer Brain Tumor and Radiotherapy Groups, National Cancer Institute of Canada Clinical Trials Group. Radiotherapy plus concomitant and adjuvant temozolomide for glioblastoma. N Engl J Med. 2005; 352(10): 987-996, doi: 10.1056/ NEJMoa043330, indexed in Pubmed: 15758009.

25. Ostrom QT, Rubin JB, Lathia JD, et al. Females have the survival advantage in glioblastoma. Neuro Oncol. 2018; 20(4): 576-577, doi: 10.1093/neuonc/noy002, indexed in Pubmed: 29474647.

26. Weller M, van den Bent M, Tonn JC, et al. European Association for Neuro-Oncology (EANO) Task Force on Gliomas. European Association for Neuro-Oncology (EANO) guideline on the diagnosis and treatment of adult astrocytic and oligodendroglial gliomas. Lancet Oncol. 2017; 18(6): e315-e329, doi: 10.1016/S1470-2045(17)30194-8, indexed in Pubmed: 28483413.

27. Giladi M, Schneiderman RS, Voloshin T, et al. Mitotic Spindle Disruption by Alternating Electric Fields Leads to Improper Chromosome Segregation and Mitotic Catastrophe in Cancer Cells. Sci Rep. 2015; 5: 18046, doi: 10.1038/srep18046, indexed in Pubmed: 26658786.

28. Stupp R, Taillibert S, Kanner A, et al. Effect of Tumor-Treating Fields Plus Maintenance Temozolomide vs Maintenance Temozolomide Alone on Survival in Patients With Glioblastoma: A Randomized Clinical Trial. JAMA. 2017; 318(23): 2306-2316, doi: 10.1001/ jama.2017.18718, indexed in Pubmed: 29260225.

29. Pham TM, Sikdar KC, Cheung WY, et al. Premature Mortality Due to Malignancies of the Central Nervous System in Canada, 1980-2010. Neuroepidemiology. 2018; 50(3-4): 195-200, doi: 10.1159/000488145, indexed in Pubmed: 29694962.

30. Bianco J, Bastiancich C, Jankovski A, et al. On glioblastoma and the search for a cure: where do we stand? Cell Mol Life Sci. 2017; 74(13): 2451-2466, doi: 10.1007/s00018-017-2483-3, indexed in Pubmed: 28210785
31. Aldape K, Zadeh G, Mansouri S, et al. Glioblastoma: pathology, molecular mechanisms and markers. Acta Neuropathol. 2015; 129(6): 829-848, doi: 10.1007/s00401-015-1432-1, indexed in Pubmed: 25943888.

32. Louis DN, Perry A, Reifenberger G, et al. The 2016 World Health Organization Classification of Tumors of the Central Nervous System: a summary. Acta Neuropathol. 2016; 131(6): 803-820, doi: 10.1007/ s00401-016-1545-1, indexed in Pubmed: 27157931.

33. Paolillo M, Boselli C, Schinelli S. Glioblastoma under Siege: An Overview of Current Therapeutic Strategies. Brain Sci. 2018; 8(1), doi: 10.3390/brainsci8010015, indexed in Pubmed: 29337870.

34. Cihoric N, Tsikkinis A, Minniti G, et al. Current status and perspectives of interventional clinical trials for glioblastoma - analysis of ClinicalTrials.gov. Radiat Oncol. 2017; 12(1): 1, doi: 10.1186/s13014-0160740-5, indexed in Pubmed: 28049492.

35. Vanderbeek AM, Rahman R, Fell G, et al. The clinical trials landscape for glioblastoma: is it adequate to develop new treatments? Neuro Oncol. 2018; 20(8): 1034-1043, doi: 10.1093/neuonc/noy027, indexed in Pubmed: 29518210.

36. Zanders ED, Svensson F, Bailey DS. Therapy for glioblastoma: is it working? Drug Discov Today. 2019; 24(5): 1193-1201, doi: 10.1016/j. drudis.2019.03.008, indexed in Pubmed: 30878561.

37. Nieder C, Astner ST, Grosu AL. Glioblastoma research 2006-2010: pattern of citation and systematic review of highly cited articles. Clin Neurol Neurosurg. 2012; 114(9): 1207-1210, doi: 10.1016/j.clineuro.2012.03.049, indexed in Pubmed: 22516416.

38. Bastian H, Glasziou P, Chalmers I. Seventy-five trials and eleven systematic reviews a day: how will we ever keep up? PLoS Med. 2010; 7(9): e1000326, doi: 10.1371/journal.pmed.1000326, indexed in Pubmed: 20877712.

39. United States National Library of Medicine. https://www.ncbi.nlm. nih.gov/pubmed.

40. National Cancer Institute Thesaurus. ncit.nci.nih.gov/ncitbrowser (2019 Aug 15).

41. Springer. AdisInsight. https://adisinsight.springer.com ( 2019 Sep 15).

42. Falagas ME, Pitsouni El, Malietzis GA, et al. Comparison of PubMed, Scopus, Web of Science, and Google Scholar: strengths and weaknesses. FASEB J. 2008; 22(2): 338-342, doi: 10.1096/fj.07-9492LSF, indexed in Pubmed: 17884971.

43. Elsevier. Scopus. https://www.scopus.com.

44. Vito MRM. segmented: an R Package to Fit Regression Models with Broken-Line Relationships. http://cran.r-project.org/doc/Rnews/.

45. Altman DG, Bland JM. How to obtain the confidence interval from a Pvalue. BMJ. 2011; 343: d2090, doi: 10.1136/bmj.d2090, indexed in Pubmed: 21824904.

46. Rich MW. Impact Factor Revisited. J Card Fail. 2017; 23(8): 579-580, doi: 10.1016/j.cardfail.2017.06.006, indexed in Pubmed: 28663098.

47. Hirsch BR, Califf RM, Cheng SK, et al. Characteristics of oncology clinical trials: insights from a systematic analysis of ClinicalTrials.gov. JAMA Intern Med. 2013; 173(11): 972-979, doi: 10.1001/jamainternmed.2013.627, indexed in Pubmed: 23699837.

48. Cohen JF, Korevaar DA, Wang J, et al. Should we search Chinese biomedical databases when performing systematic reviews? Syst Rev. 2015; 4 : 23, doi: 10.1186/s13643-015-0017-3, indexed in Pubmed: 25874584. 